\title{
SURGICAL VERSUS ULTRASOUND GUIDED ASPIRATION OR DRAINAGE OF DEEP NECK SPACE ABSCESSES: A RANDOMIZED CONTROLLED TRIAL
}

Alaa Hazem Gaafar, *Mohamed Mahmoud Elshafei, Mostafa Magdy Donia, Abdelrahman Mostafa Hassan Department of Otorhinolaryngology, ${ }^{*}$ Department of Diagnostic \& Interventional Radiology, Faculty of Medicine, University of Alexandria.

\section{Introduction}

Deep neck space abscesses are considered to be one of life-threatening emergencies the head and neck region. Traditionally, the main treatment for deep neck space abscesses was surgical drainage with sufficient antimicrobial treatment. Intraoral or external approaches can be used for surgical drainage of neck abscesses. Despite the effectiveness of these procedures, they have some remarkable disadvantages. General anesthesia is required, which adds a considerable risk for patients especially those with bad general condition. Intraoral approaches have their limitations like poor visualization and airway compromise. External approaches usually necessitate neck incisions and exploration, which exposes patients to the risk of injury to vital neurovascular structures and a cosmetically unpleasant scar.

Recent literature suggested a less invasive and an effective alternative to surgical incision and drainage by performing ultrasound-guided drainage of neck abscesses. It abolishes most of the drawbacks of incision and drainage and has been proven to be effective in certain cases.

\section{Aim of the work}

The aim of this study is to compare surgical drainage versus ultrasound-guided aspiration or drainage of well-defined deep neck space abscesses, using a randomized controlled clinical trial design.

\section{Patients}

\section{Inclusion criteria}

Evidence of a well-defined deep neck space abscess on contrast-enhanced computed tomography scan (CECT).

Exclusion criteria

Patients with airway compromise.

Retropharyngeal abscesses.

Deep neck space abscess associated with neck neoplasm.

Deep neck space abscess complicated with necrotizing fasciitis.

\section{METHODS}

Each patient was subjected to the following:

1. History taking.

2.Physical examination.

Neck examination.

Airway assessment

Dental work up.

3.Laboratory evaluation:

Routine laboratory investigations.

4. Radiological evaluation

Computed tomography with contrast enhancement (CECT).

Ultrasound neck.

Panoramic dental X-ray was obtained in cases with suspected dental source

of infection.

Treatment approach

1) Antibiotics:

A-Empirical broad-spectrum antibiotics.

B-Culture directed antibiotics:

Pus obtained during intervention either by ultrasound guided aspiration or

surgery was sent immediately for culture and drug sensitivity.

2) Procedure: Patients who met the inclusion criteria with a well-defined neck space abscess were recruited in the study and block-randomized to two

groups:

Group A: Ultrasound-guidedaspiration.

Group B: Surgical incision and drainage.

\section{Results}

Table (1): Comparison between the two studied groups according to scar formation

\begin{tabular}{|c|c|c|c|c|c|}
\hline \multirow[t]{2}{*}{ Scar formation } & \multicolumn{2}{|c|}{$(\mathbf{n}=\mathbf{1 5})$} & \multicolumn{2}{|c|}{$(\mathbf{n}=\mathbf{1 5})$} & \multirow[t]{2}{*}{$\mathbf{p}$} \\
\hline & No. & $\%$ & No. & $\%$ & \\
\hline No & 15 & 93.8 & $\begin{array}{c}0 \\
16\end{array}$ & 0.0 & $<0.001^{\prime \prime}$ \\
\hline
\end{tabular}

$\mathrm{p}$ : $\mathrm{p}$ value for Chi square test for comparing between the studied groups *: Statistically significant at $\mathrm{p} \leq 0.05$
Table (2): Comparison between the two studied groups according to need of $2^{\text {nd }}$ drainage

\begin{tabular}{|l|c|c|c|c|c|}
\hline \multirow{2}{*}{ Need of 2 $2^{\text {nd }}$ drainage } & \multicolumn{2}{|c|}{$\begin{array}{c}\text { Group A } \\
(\mathbf{n = 1 6})\end{array}$} & \multicolumn{2}{c|}{$\begin{array}{c}\text { Group B } \\
(\mathbf{n = 1 6})\end{array}$} & \multirow{2}{*}{${ }^{\mathbf{F E}} \mathbf{p}$} \\
\cline { 2 - 5 } & No. & \% & No. & $\%$ & \\
\hline No & 12 & 75.0 & 14 & 87.5 & \multirow{2}{*}{} \\
Yes & 4 & 25.0 & 2 & 12.5 & 0.654 \\
\hline
\end{tabular}

FE: Fisher Exact p: p value for comparing between the studied groups

Table (3): Comparison between the two studied groups according to length of hospital stay in days

\begin{tabular}{|l|c|c|c|c|}
\hline $\begin{array}{c}\text { Length of } \\
\text { hospital stay }\end{array}$ & $\begin{array}{c}\text { Group A } \\
(\mathbf{n = 1 6})\end{array}$ & $\begin{array}{c}\text { Group B } \\
(\mathbf{n}=\mathbf{1 6})\end{array}$ & $\mathbf{U}$ & $\mathbf{p}$ \\
\hline Min. - Max. & $1.0-5.0$ & $3.0-7.0$ & & \\
Mean \pm SD. & $2.63 \pm 1.54$ & $4.81 \pm 1.47$ & $43.0^{*}$ & $0.001^{*}$ \\
Median (IQR) & $3.0(1.0-3.50)$ & $4.50(4.0-6.0)$ & & \\
\hline
\end{tabular}

\section{U: Mann Whitney test $\begin{aligned} & \text { IQR: Inter quartile range } \\ & \text { W }\end{aligned}$ \\ $p: p$ value for comparing between the studied groups
*: Statistically significant at $\mathrm{p} \leq 0.05$}

\section{Conclusion}

Ultrasound-guided aspiration is a safe and an effective alternative to surgical incision and drainage in unilocular or multilocular deep neck space abscesses. In addition, it is associated with shorter hospital stay and thus may reduce healthcare costs.

There must be good coordination between the interventional radiologist and otolaryngologist especially in judging whether further needle aspiration would be safe or open surgical incision and drainage is needed.

We recommend a trial of Ultrasound-guided aspiration in all cases of deep neck space abscesses in case of absence of airway compromise, necrotizing fasciitis or retropharyngeal space affection. 